\title{
Aspects of the FM Kondo Model: From Unbiased MC Simulations to Back-of-an-Envelope Explanations
}

\author{
Maria Daghofer ${ }^{1}$, Winfried Koller ${ }^{2}$, Alexander Prüll ${ }^{1}$, Hans Gerd Evertz ${ }^{1}$, \\ and Wolfgang von der Linden ${ }^{1}$ \\ 1 Institute for Theoretical and Computational Physics \\ Graz University of Technology \\ daghofer@itp.tu-graz.ac.at \\ 2 Department of Mathematics, Imperial College
}

Summary. Effective models are derived from the ferromagnetic Kondo lattice model with classical corespins, which greatly reduce the numerical effort. Results for these models are presented. They indicate that double exchange gives the correct order of magnitude and the correct doping dependence of the Curie temperature. Furthermore, we find that the jump in the particle density previously interpreted as phase separation is rather explained by ferromagnetic polarons.

Manganites [1] are often described by the ferromagnetic Kondo lattice model, which is considered to explain some of their features, e.g., the transition from antiferromagnetic to ferromagnetic order with doping [2].

The application of the model is motivated by the fact, that crystal field splitting divides the five d-orbitals into two $e_{g}$ and three $t_{2 g}$ orbitals, where the latter are energetically favored in the case of manganites. All three $t_{2 g}$ orbitals are singly occupied and rather localized. Due to a strong Hund's rule coupling, these electrons are aligned in parallel and form a core spin with length $S=3 / 2$. The filling of the $e_{g}$ orbitals is determined by doping and these electrons can hop from one $\mathrm{Mn}$ ion to the next via the intermediate oxygen. Hund's rule coupling leads to a ferromagnetic interaction between the itinerant $e_{g}$ electrons and the $t_{2 g}$ core spin. The core spins interact through super exchange leading to a weak antiferromagnetic coupling between them.

In this chapter, we derive effective models for the ferromagnetic Kondo lattice model and introduce suitable Markov chain Monte Carlo (MC) algorithms. The presented results, were not obtainable by simple analytic considerations, are partly found by this MC method and partly by use of the Wang-Landau algorithm [3]. 


\section{Model Hamiltonian}

The spin and charge degrees of freedom in manganites can be described by the ferromagnetic Kondo lattice model with two orbitals $\left(x^{2}-y^{2}\right.$ and $\left.3 z^{2}-r^{2}\right)$ :

$$
\hat{H}=-\sum_{i, j, \alpha, \beta, \sigma} t_{i \alpha, j \beta} c_{i \alpha \sigma}^{\dagger} c_{j \beta \sigma}-\tilde{J}_{H} \sum_{i \alpha} \boldsymbol{\sigma}_{i \alpha} \cdot \boldsymbol{S}_{i}+J^{\prime} \sum_{<i j>} \boldsymbol{S}_{i} \cdot \boldsymbol{S}_{j},
$$

where $c_{i \alpha \sigma}^{\dagger}\left(c_{i \alpha \sigma}\right)$ creates (annihilates) an electron with spin $\sigma$ in orbital $\alpha$ at site $i, \boldsymbol{\sigma}_{i \alpha}$ denotes electron spin in orbital $\alpha$ and $\boldsymbol{S}_{i}$ the core spin. The first term of the Hamiltonian describes the hopping between the nearest neighbor sites; the hopping strength $t_{i \alpha, j \beta}$ depends on the involved orbitals and the direction. As matrices in the orbital indices $\alpha, \beta=1(2)$, corresponding to the $x^{2}-y^{2}\left(3 z^{2}-r^{2}\right)$ orbitals (see e.g. [2]), this hopping reads

$$
t_{i, i+\hat{z}}=t\left(\begin{array}{ll}
0 & 0 \\
0 & 1
\end{array}\right), \quad t_{i, i+\hat{x} / \hat{y}}=t\left(\begin{array}{cc}
3 / 4 & \mp \sqrt{3} / 4 \\
\mp \sqrt{3} / 4 & 1 / 4
\end{array}\right) .
$$

The overall hopping strength is $t$, which will be used as unit of energy, by setting $t=1$.

The second term contains the ferromagnetic interaction between the electrons and the core spins and the third term is the AFM superexchange of the core spins.

The $S=3 / 2$ core spin can approximately be treated as a classical spin, which corresponds to the limit $S \rightarrow \infty[4,5]$. It is then replaced by a vector of unit length and the factor $3 / 2$ is incorporated into $\tilde{J}_{H}$. This approximation simplifies calculations enormously and should not lead to much difference from the quantum case except possibly for very low temperatures $T \approx 0$ [5-9].

For large $\tilde{J}_{H}$, the electronic density of states of the Hamiltonian (1) is split into the lower and upper Kondo band, separated by approximately $\tilde{J}_{H}$. The $e_{g}$ electrons move mostly parallel to the core spins in the lower band, and anti-parallel in the upper band.

In order to derive effective low-energy models for $\tilde{J}_{H} \gg t, J^{\prime}$, we change the quantization axis for the electron spin from the global quantization axis (e.g. the z-direction) to a local quantization axis, namely the direction of the local $t_{2 g}$ core spin. Spin up (down) then means that the $e_{g}$ electron spin is parallel (antiparallel) to the core spin. The Hund's rule term $\tilde{J}_{H} \boldsymbol{\sigma}_{i \alpha} \cdot \boldsymbol{S}_{i}$ becomes $J_{H}\left(\hat{n}_{i \alpha \downarrow}-\hat{n}_{i \alpha \uparrow}\right)$, with the factor $1 / 2$ coming from the electron spin also absorbed into $J_{H}$. While the $e_{g}$-spin is preserved in global quantization, this is no longer the case in the local quantization. An up electron at site $i$ can therefore become a down electron at site $j$, which is denoted by the superscript for the hopping strength. Furthermore, the hopping now depends on the core spins:

$$
t_{i \alpha, j \beta}^{\sigma, \sigma^{\prime}}=t_{i \alpha, j \beta} u_{i j}^{\sigma, \sigma^{\prime}}
$$

The first factor is the orbital-dependent hopping strength (2) and the second factor contains the relative orientation of the core spins: 


$$
\begin{aligned}
u_{i, j}^{\sigma, \sigma} & =c_{i} c_{j}+s_{i} s_{j} \mathrm{e}^{i \sigma\left(\phi_{j}-\phi_{i}\right)}=\cos \left(\vartheta_{i j} / 2\right) \mathrm{e}^{i \psi_{i j}} \\
u_{i, j}^{\sigma,-\sigma} & =\sigma\left(c_{i} s_{j} e^{-i \sigma \phi_{j}}-c_{j} s_{i} e^{-i \sigma \phi_{i}}\right)=\sin \left(\vartheta_{i j} / 2\right) \mathrm{e}^{i \chi_{i j}}
\end{aligned}
$$

with the abbreviations $c_{j}=\cos \left(\theta_{j} / 2\right)$ and $s_{j}=\sin \left(\theta_{j} / 2\right)$ and the restriction $0 \leq \theta_{j} \leq \pi$, where $\theta_{i}, \phi_{i}$ are the polar coordinates for core spin $\boldsymbol{S}_{i}$. These factors depend on the relative angle $\vartheta_{i j}$ of the core spins $\boldsymbol{S}_{i}$ and $\boldsymbol{S}_{j}$ and on some complex phases $\psi_{i j}$ and $\chi_{i j}$. With a shift of the chemical potential $\mu \rightarrow \mu-J_{H}$, the Hamiltonian (1) in local spin-quantization reads:

$$
\hat{H}=-\sum_{i, j, \alpha, \beta, \sigma, \sigma^{\prime}} t_{i \alpha, j \beta}^{\sigma, \sigma^{\prime}} c_{i \alpha \sigma}^{\dagger} c_{j \beta \sigma^{\prime}}+2 J_{H} \sum_{i \alpha} \hat{n}_{i \alpha \downarrow}+J^{\prime} \sum_{<i j>} \boldsymbol{S}_{i} \cdot \boldsymbol{S}_{j}
$$

This is still the same Hamiltonian as (1) without any approximation besides the use of classical core spins.

\subsection{Effective Spinless Fermions}

Most of the experimental results on manganites and all of the theoretical work presented here concerns electron densities $0 \leq n_{\mathrm{el}} \leq 1$, i. e. predominantly the lower Kondo band. As $J_{H}$ is much larger than the hopping $t$ and the AFM superexchange $J^{\prime}$, one can simplify the model by a separation of energy scales [10]. As a first approximation, one can take $J_{H} \rightarrow \infty$ and thereby leave out the configurations with $e_{g}$ electrons antiparallel to the core spins completely. This approximation is widely used $[11,12]$, but misses some important effects discussed in Sect. 3.1. However, if one treats these configurations in second order perturbation theory $[13,14]$, almost perfect agreement to the original Kondo lattice model is obtained without any additional numerical effort [15]. This approach is similar to the derivation of the $t-J$ model from the Hubbard model, while the $J_{H} \rightarrow \infty$ method corresponds to $U \rightarrow \infty$ for the Hubbard model.

In this effective model, the dynamical degrees of freedom are the low energy states with the $e_{g}$-spins parallel (i. e. up in local quantization) to the $t_{2 g}$-spins. The virtual excitations meditated by the hopping matrix are configurations where one $e_{g}$ electron is antiparallel (down):

$(i \alpha \uparrow) \rightarrow(j \beta \downarrow) \rightarrow\left(i^{\prime} \alpha^{\prime} \uparrow\right)$. As the low energy states contain only up electrons, this lead to an effective spinless fermion Hamiltonian:

$$
\begin{aligned}
\hat{H}_{\mathrm{ESF}}= & -\sum_{i, j, \alpha, \beta} t_{i \alpha, j \beta}^{\uparrow \uparrow} c_{i \alpha}^{\dagger} c_{j \beta}-\sum_{i, \alpha, \alpha^{\prime}}\left(\sum_{j, \beta} \frac{t_{i \alpha^{\prime}, j \beta}^{\uparrow \downarrow} t_{j \beta, i \alpha}^{\downarrow \uparrow}}{2 J_{H}}\right) c_{i \alpha^{\prime}}^{\dagger} c_{i \alpha} \\
& -\sum_{\left[i \neq i^{\prime}\right], \alpha, \alpha^{\prime}}\left(\sum_{j, \beta} \frac{t_{i^{\prime} \alpha^{\prime}, j \beta}^{\uparrow \downarrow} t_{j \beta, i \alpha}^{\downarrow \uparrow}}{2 J_{H}}\right) c_{i^{\prime} \alpha^{\prime}}^{\dagger} c_{i \alpha}+J^{\prime} \sum_{<i j>} \boldsymbol{S}_{i} \cdot \boldsymbol{S}_{j} .
\end{aligned}
$$

The first term of this Hamiltonian contains the kinetic energy of the electrons

moving in the lower Kondo band. As $t_{i \alpha, j \beta}^{\uparrow \uparrow}$ is largest for parallel core spins, this term favors ferromagnetism. The second term describes electrons that 
get excited into the upper Kondo band and then hop back to the original site. It yields a density dependent antiferromagnetic interaction between the core spins. The third term is a 'three-site-term' of minor influence and will be neglected [15]. On the other hand, its inclusion does not increase the numerical effort.

The reduction of the Hilbert space achieved by this effective model is the same as for the $J_{H} \rightarrow \infty$ limit, and finite $J_{H}$ can thus be treated with the same numerical effort.

\subsection{Uniform Hopping Approach}

A significant further simplification is the uniform hopping approximation proposed by van den Brink and Khomskii [16]. This approximation replaces the different angles of neighboring core spins by a mean value. In order to treat anisotropies, two different angles are chosen, $\theta_{z}=\boldsymbol{S}_{i} \cdot \boldsymbol{S}_{i \pm z}$ in z-direction and $\theta_{x y}=\boldsymbol{S}_{i} \cdot \boldsymbol{S}_{i \pm x}=\boldsymbol{S}_{i} \cdot \boldsymbol{S}_{i \pm y}$ within the $x y$-plane. These should not be confused with the polar angle of an individual core spin $\theta_{i}$. It is assumed that the relative orientation is the same between all nearest neighbor pairs. The hopping matrix therefore becomes translationally invariant. Spin configurations that are still treated exactly include, among others, ferro- and antiferromagnetism and spin canted states.

The impact of the core spins on the hopping simplifies to

$$
u_{z}^{\sigma, \sigma}=\cos \left(\frac{\theta_{z}}{2}\right)=u_{z} \quad, \quad u_{z}^{\sigma,-\sigma}=\sin \left(\frac{\theta_{z}}{2}\right)=\sqrt{1-u_{z}^{2}}
$$

in z-direction and analogously in $\mathrm{x} / \mathrm{y}$-direction. Likewise, the inner product of the $t_{2 g}$ spins entering the superexchange term can be expressed by

$$
\mathbf{S}_{i} \cdot \mathbf{S}_{i+\hat{z}}=\cos \theta_{z}=2 u_{z}^{2}-1 .
$$

The energy of this model can easily be evaluated, especially in the thermodynamic limit and the ground state can be obtained by minimizing the energy with respect to $\theta_{z}$ and $\theta_{x y}$.

For a one orbital model in one dimension with periodic boundary conditions, the Hamiltonian simplifies to

$$
\hat{H}=-u_{z} \sum_{\langle i j\rangle} c_{i}^{\dagger} c_{j}-\frac{1-u_{z}^{2}}{J_{H}} \sum_{i} c_{i}^{\dagger} c_{i}+J^{\prime} L\left(2 u_{z}^{2}-1\right),
$$

This Hamiltonian yields a shifted tight-binding band structure

$$
\epsilon_{k}=-2 u \cos (k)-\left(1-u_{z}^{2}\right) / J_{H}
$$

with a band width of $4 u_{z}$, which has its maximum for ferromagnetic core spins and vanishes for antiferromagnetic order. 
Similar calculations can be done for three dimensions with both orbitals and a ground state phase diagram can thus be obtained, see $[15,16]$.

This UHA approach was extended to finite temperatures in [17]. To introduce this method, we proceed as follows: For a given core spin configuration $\mathcal{S}$, characterized by the set of angles $\left\{\theta_{i}, \phi_{i}\right\}$, we define the average $u$-value

$$
u(\mathcal{S})=\frac{1}{N_{p}} \sum_{\langle i j\rangle} u_{i j}^{\uparrow \uparrow}(\mathcal{S}) .
$$

Here $N_{p}$ is the number of n.n. pairs $\langle i j\rangle$. In the ESF Hamiltonian (7), $u_{i j}^{\uparrow \uparrow}$ is then replaced by $u(\mathcal{S})$. Besides $u_{i j}^{\uparrow \uparrow}$ the Hamiltonian depends on $\left|u_{i j}^{\sigma,-\sigma}\right|^{2}$ and on $\mathbf{S}_{i} \cdot \mathbf{S}_{j}$, which correspond to $\sin ^{2}\left(\vartheta_{i j} / 2\right)$ and $\cos \vartheta_{i j}$, respectively. As a further approximation, these terms are replaced by $1-u^{2}(\mathcal{S})$ and $2 u^{2}(\mathcal{S})-1$ respectively, which leads to the one-orbital UHA Hamiltonian

$$
\hat{H}=-u \sum_{\langle i j\rangle} c_{i}^{\dagger} c_{j}-\frac{1-u^{2}}{2 J_{H}} \sum_{i} z_{i} c_{i}^{\dagger} c_{i}+J^{\prime} N_{p}\left(2 u^{2}-1\right),
$$

with $z_{i}$ being the number of nearest neighbors for site $i$. This Hamiltonian defines the Boltzmann factor for the spin configuration $\mathcal{S}$. In order to calculate thermodynamical expectation values, one still needs to calculate the density of states $\Gamma(u)$, i.e. the number of spin configurations with the same average value for $u$. It can be calculated exactly in one dimension and by use of the Wang-Landau algorithm [3] in higher dimensions.

Once the density of states $\Gamma(u)$ has been obtained, observables can be obtained for any temperature, much larger lattices can be treated and a 3D phase diagram for finite temperatures can be obtained. The numerical effort is reduced from an integration over the $L$-dimensional space of the core spin configurations to an integral over the one-dimensional unit interval for $u$.

\section{Monte Carlo Algorithm}

The algorithm used to simulate the Kondo lattice model and the effective spinless fermion model in the grand canonical ensemble is the one proposed in [18]. For each core spin configuration, the resulting Hamiltonian for the $e_{g}$ electrons is a one-particle problem. The statistical weight for the core-spin configuration $\mathcal{S}$ in the grand canonical ensemble is the starting point of grand canonical Markov chain Monte Carlo simulations:

$$
w(\mathcal{S} \mid \mu)=\frac{\operatorname{tr}_{c} \mathrm{e}^{-\beta(\hat{H}(\mathcal{S})-\mu \hat{N})}}{\mathcal{Z}(\mu)} .
$$

It is calculated from the eigenvalues of $\hat{H}(\mathcal{S})$ by use of free fermion formulae, which is denoted by the trace over the fermionic degrees of freedom $\operatorname{tr}_{c}$. 
As some particle numbers are not stable in the grand canonical ensemble, we developed a canonical algorithm. An exact approach would mean calculating the Boltzmann weight for every possible distribution of $N_{\mathrm{el}}$ electrons on $L$ eigenvalues and summing over these contributions. This is numerically too demanding. But for low temperatures, only very few of these distributions actually contribute to the partition function. They can be obtained by filling $N_{0}<N_{\text {el }}$ electrons into the $N_{0}$ lowest eigenenergies and distributing only the remaining $N_{\mathrm{el}}-N_{0} \approx 5$ electrons on the states around the Fermi-energy. The canonical weight then becomes:

$$
w\left(\mathcal{S} \mid N_{e l}\right)=\frac{\sum_{\tilde{\mathcal{P}}} \mathrm{e}^{-\beta \hat{H}\left(\mathcal{S}, \tilde{\mathcal{P}}\left(N_{e l}\right)\right)}}{\mathcal{Z}\left(N_{e l}\right)},
$$

where $\tilde{\mathcal{P}}$ denotes these restricted permutations. In order to decrease autocorrelations, particle fluctuations within a set of 3 to 5 densities were allowed. The amount of core-spin rotations was small for most updates in order to ensure high acceptance, but occasionally a complete spin flip was proposed. In one dimension, whole sections of the chain were rotated at once. 50 to several hundreds of sweeps were skipped between measurements. This ensured statistical independence for the 1D calculations, in 2D, remaining autocorrelations were treated by autocorrelation analysis.

While observables depending only on $\mathcal{Z}$ are independent of the spin quantization (global/local), care must be taken when evaluating e.g. the one particle Greens function, which in global quantization can be written as

$$
\sum_{\sigma} \ll a_{i \sigma} ; a_{j \sigma}^{\dagger} \gg_{\omega}=\int \mathcal{D}[\mathcal{S}] w(\mathcal{S} \mid \mu) u_{j i}^{\uparrow \uparrow}(\mathcal{S}) \ll c_{i} ; c_{j}^{\dagger} \gg_{\omega}^{\mathcal{S}},
$$

where $\ll c_{i} ; c_{j}^{\dagger} \gg_{\omega}^{\mathcal{S}}$ is the Green's function in local spin quantization. It can be expressed in terms of the one-particle eigenvalues $\epsilon^{(\lambda)}$ and the corresponding eigenvectors $\psi^{(\lambda)}$ of the Hamiltonian $\hat{H}(\mathcal{S})$ :

$$
\ll c_{i} ; c_{j}^{\dagger} \gg_{\omega}^{\mathcal{S}}=\sum_{\lambda} \frac{\psi^{(\lambda)}(i) \psi^{*(\lambda)}(j)}{\omega-\left(\epsilon^{(\lambda)}-\mu\right)+i 0^{+}}
$$

It should be pointed out that the one-particle density of states (DOS) is identical in global and local quantization; for details see [17].

\section{Results}

In the first subsection, we will demonstrate the validity of the simplified effective models. We will then use the uniform hopping approach (UHA) in Sect. 3.2 to determine the Curie temperature of the one-orbital model in 
three dimensions. In Sect. 3.3, we will present results for the one- and twodimensional model and we will show that they do not indicate phase separation, but rather ferromagnetic polarons. A phase diagram for the 2D model is given in Sect. 3.4.

\subsection{Validity of the ESF Model and the UHA in one Dimension}

In this section, we will present results from unbiased Monte Carlo Simulations for the ESF model in one dimension with one orbital and open boundary conditions and we will compare them to results for the full ferromagnetic Kondo lattice model and the $J_{H} \rightarrow \infty$ approximation. Simulations were done for $L=20$ sites, $\beta=50, J^{\prime}=0.02$ and $J_{H}$ varying from 4 to 10 .

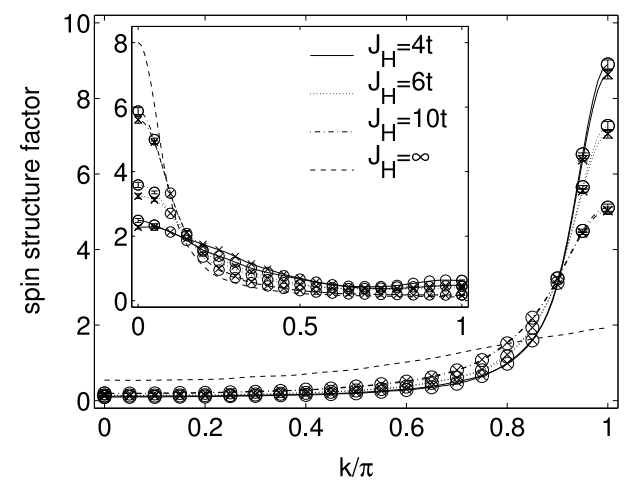

Fig. 1. Spin structure factor for the $t_{2 g}$ spins at $n=1$ (inset: $n \approx 0.75$ ) for $\beta=50, J^{\prime}=0.02, L=20$ and different values of $J_{H}$. Circles: spinless fermion model (7); crosses: DE model (6). In the limit $J_{H} \rightarrow \infty$ (dashed line) the intensity of the AFM peak is considerably smaller than for finite $J_{H}$. From Ref. [15].

Figure 1 shows the core-spin structure factor for the three models. For electron density $n \approx 0.75$ (inset), the $t_{2 g}$ correlations are ferromagnetic, driven by the kinetic energy of the $e_{g}$ electrons. For $n=1$, the lower Kondo band is completely filled, no hopping is possible and the kinetic energy therefore vanishes. Excitations into the upper Kondo band (virtual for the ESF), which are favored by antiferromagnetism, then dominate the energy.

For both densities and even for moderate $J_{H}=4$, the ESF model (7) and the original Kondo Model (6) produce virtually identical results. The $J_{H} \rightarrow \infty$ model on the other hand does not reproduce the antiferromagnetic correlations for the completely filled lower Kondo band correctly, because the virtual excitations are missing from this model, and it also overestimates the ferromagnetic correlations at $n \approx 0.75$. The AFM effect coming from the virtual excitations can be described by a density dependent effective parameter 
$J_{\text {eff }}=J^{\prime}+1 /\left(2 J_{H}\right)$ at $n=1$ and it is generally much stronger than the small superexchange $J^{\prime}$ also favoring AFM.

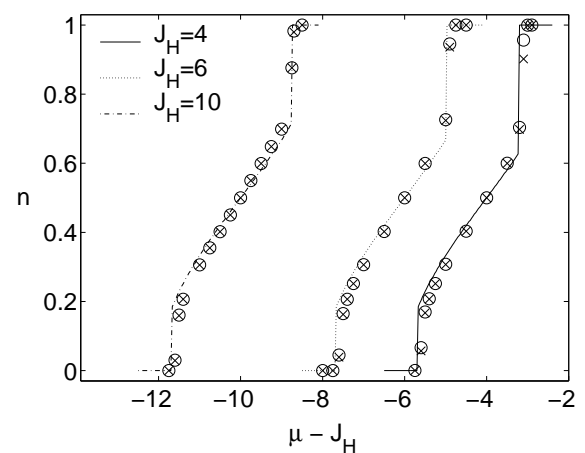

Fig. 2. Electron density versus chemical potential for $J_{H}=4,6$, and 10 (right to left), and $J^{\prime}=0.02$. MC results at $\beta=50, L=20$ for the spinless fermion model $H_{\mathrm{ESF}}$ (circles) are compared with those for the DE model $H$ (crosses). Error bars of the MC data are smaller than the symbols. The lines correspond to groundstate UHA. From Ref. [15].

Figure 2 shows the electron density versus the chemical potential $\mu$ for the Kondo model, the effective spinless fermions and groundstate UHA for $J_{H}=4,6,10$. All three models give almost identical results. For very small $\mu$, the band is empty and $J^{\prime}$ leads to antiferromagnetism. At a critical $\mu_{1}^{c}$ depending on $J_{\text {eff }}=J^{\prime}$, the filling jumps to $n \approx 0.2$ and the correlations become ferromagnetic. At a second critical $\mu_{2}^{c}$, depending on $J_{\text {eff }}=J^{\prime}+$ $1 /\left(2 J_{H}\right)$, it becomes antiferromagnetic again and the density jumps from $n \approx$ 0.7 to $n \approx 1$.

These discontinuities mean that intermediate particle numbers are not stable in the grand canonical ensemble. They have been interpreted as phase separation. However, we will show in Sect. 3.3 that their cause lies rather in small ferromagnetic polarons.

\subsection{Finite Temperature UHA and Curie Temperature}

Although the uniform hopping approach replaces the fluctuating core spins by an average $u$, it reproduces not only the expectation value of the energy, but also its width with astonishing accuracy, even for higher temperatures. The results of UHA also remain valid upon the inclusion of n.n Coulomb repulsion [17]. All the while, the numerical effort is reduced from sampling over hundreds of thousands of core-spin configurations to scanning the single parameter $u$ within the unit interval. The fact that the results remain valid with inclusion of the Coulomb repulsion indicates that UHA is a reli- 
able approximative method that can safely be extended to more complicated situations.

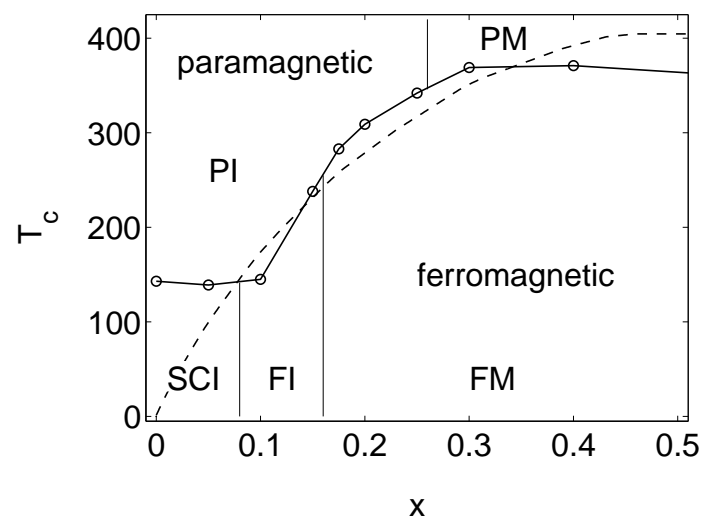

Fig. 3. Curie temperature (dashed line) of the one-orbital DE model for a $16^{3}$ cluster and $t=0.2 \mathrm{eV}$ calculated in UHA. Circles and phases PM (paramagnetic metal), PI (paramagnetic insulator), FM (ferromagnetic metal), FI (ferromagnetic insulator), and SCI (spin canted insulator) are experimental results for $\mathrm{La}_{1-x} \mathrm{Sr}_{x} \mathrm{MnO}_{3}$ [19], UHA results [17].

As observables can be evaluated for all temperatures once the density of states $\Gamma(u)$ is known, it can be used to determine the Curie temperature for the three dimensional one-orbital model with $J_{H}=\infty$ and $J^{\prime}=0$. If one sets the only free parameter, namely the hopping strength $t$, which was also used as unit of energy, to $t=0.2 \mathrm{eV}$, in accordance with experiments, one obtains the Curie temperature in reasonable agreement with experiment, see Fig. 3. In order to obtain the different low temperature phases besides FM and PM observed in experiments for low carrier concentrations, finite $J_{H}$ and $J^{\prime}$ would be needed as well as two orbitals with Coulomb repulsion.

\subsection{Phase Separation versus Ferromagnetic Polarons}

The discontinuity of the filling as a function of the chemical potential, see Fig. 2 , is usually interpreted as phase separation [18], i.e. the system is expected to split into antiferromagnetic domains with low and ferromagnetic domains with higher carrier concentration. Taking Coulomb interactions into account, PS has been argued to lead to either small [20] or large [21] (nano-scale) clusters, which have been the basis for a possible though controversial [22] explanation of CMR [2,23]. More thorough evaluation of the MC data for the transition near the filled lower Kondo band reveals however, that single hole ferromagnetic polarons are stabilized instead, even without any Coulomb 
repulsion. All results presented in this section are for the effective spinless fermion model (7).
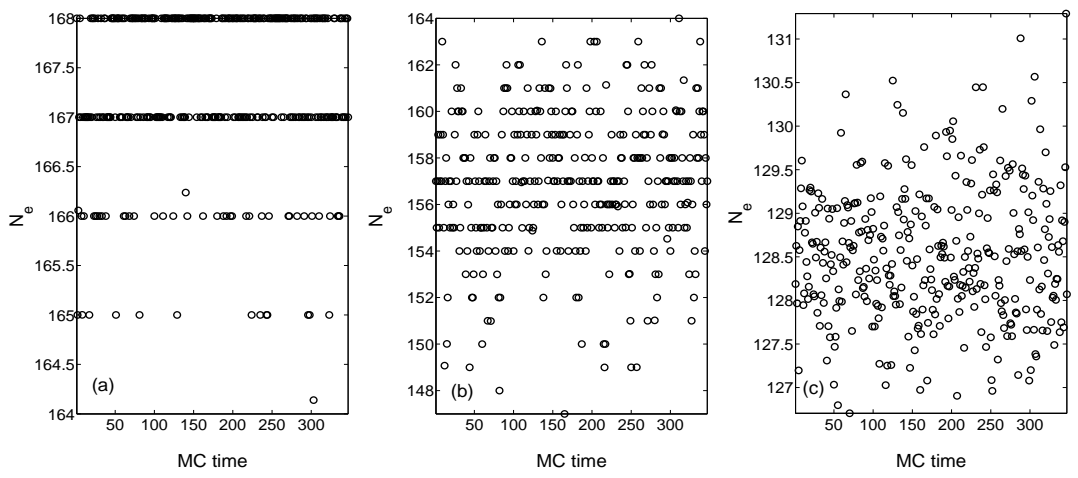

Fig. 4. Mean particle numbers $N_{\mathrm{e}}$ in a grand canonical MC simulation in 2 dimensions $\left(L=14 \times 12, J_{H}=6, J^{\prime}=0.02, \beta=50\right)$ as a function of MC time. One time step corresponds to 200 sweeps of the lattice. (a) $\mu=1.26>\mu^{*}$ : almost filled, (b) $\mu=1.19 \simeq \mu^{*}$ : polaron regime, (c) $\mu=1.12<\mu^{*}$ : FM regime. For visibility, only the first 350 time steps are shown.

A first indication for ferromagnetic polarons is the behavior of the electron density $\langle n\rangle_{\mathcal{S}}$ with the MC time near the critical chemical potential as depicted in Fig. 4, where $\langle n\rangle_{\mathcal{S}}$ is the thermodynamical expectation value for the filling given the core-spin configuration $\mathcal{S}$ sampled by the MC run. In the FM regime [Fig.4(c)], the density fluctuates slightly with the fluctuating core-spin configurations and takes non-integer values in accordance with standard results for free electrons. For the almost filled band [Fig.4(a)], the density is $\langle n\rangle_{\mathcal{S}}=1$ (0 holes) for most spin configurations and occasionally, configurations occur, which contain exactly one, exactly two or exactly three holes. In between [Fig.4(b)], the particle number fluctuates strongly, but as for $\langle n\rangle_{\mathcal{S}} \approx 1$, almost only integer fillings occur. While these integer fillings can hardly be understood in a PS scenario which is supposed to be a mixture of the low- and the high-density phases, it can easily be explained by independent polarons containing one hole each. As they are independent from each other, all have the same energy exactly balanced by the critical chemical potential $\mu^{*}$ and their number therefore fluctuates strongly.

To measure the size of the ferromagnetic domains, we use the dressed core-spin correlation function

$$
S_{h}(l)=\frac{1}{L-l} \sum_{i=1}^{L-l} n_{i}^{h} \boldsymbol{S}_{i} \cdot \boldsymbol{S}_{i+l}
$$


that measures the correlations around a hole. The hole- density operator $n_{i}^{h}$ is related to the electron density via $n_{i}^{h}=1-n_{i}$. Equation (18) holds for open boundary conditions (employed in 1D), the formula for periodic boundary conditions used in $2 \mathrm{D}$ is analogous.
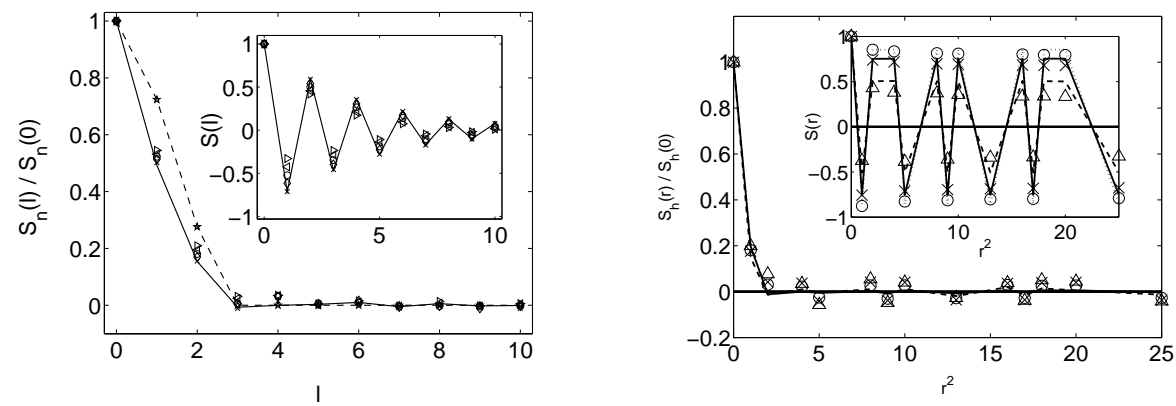

Fig. 5. Dressed spin-spin correlation function (18) from unbiased MC for $\beta=50$, $J^{\prime}=0.02$, and $J_{H}=6$. Left panel: 1 dimension: $L=50$-site chain containing one $(\times)$, two $(\diamond)$, three $(\circ)$, four $(\triangleleft)$, and five $(\triangleright)$ holes. The dashed line is calculated within the simple polaron picture, while the solid line represents the generalized UHA result for a single polaron, see [24]. Right panel: 2 dimensions: $12 \times 14$ lattice with $1(\circ), 6(\times)$ and $20(\triangle)$ holes. Continuous lines are data for the simple Polaron model (see Fig. 6, right panel): 1 Polaron (dotted), 6 (solid) and 20 Polarons (dashed). The insets show the conventional spin-spin correlation function $S(l)=\frac{1}{L-l} \sum_{i=1}^{L-l} \boldsymbol{S}_{i} \cdot \boldsymbol{S}_{i+l}$. Left from Ref. [24], right from Ref. [25].

Figure 5 shows this dressed core-spin correlation. The ferromagnetic regions around the holes are small and their size does not grow with doping neither for the one- nor for the the two-dimensional case. This indicates that the introduction of more holes leads to more small FM polarons rather than to a growth of the existing ones.

These observations lead to the development of a simple toy model for the FM polarons. Each polaron consist of a small (3-4 sites in 1D, 5 sites in 2D) FM well, in which the hole can delocalize. These wells are embedded into an AFM background. For a schematic representation, see Fig. 6. The value of the critical chemical potential can be obtained from the toy model by simple energy considerations. It is simply the difference between the energy gained by the delocalized hole and the energy payed for the breaking of AFM bonds. In this simplest model, the hopping strength is given by $u_{f}=1$ for the FM regions and $u_{a}=0$ for AFM bonds. The impact of thermal fluctuations of the core spins can be modeled by a generalization of UHA to inhomogeneous structures, where $\Gamma\left(u_{f}, u_{a}\right)$ has to be determined.

This was done for the one-dimensional model, for results and details of the algorithm see [24]. In order to compare the toy model to the MC data in 2 dimensions, random deviations were added to the core spins, see [25]. The 

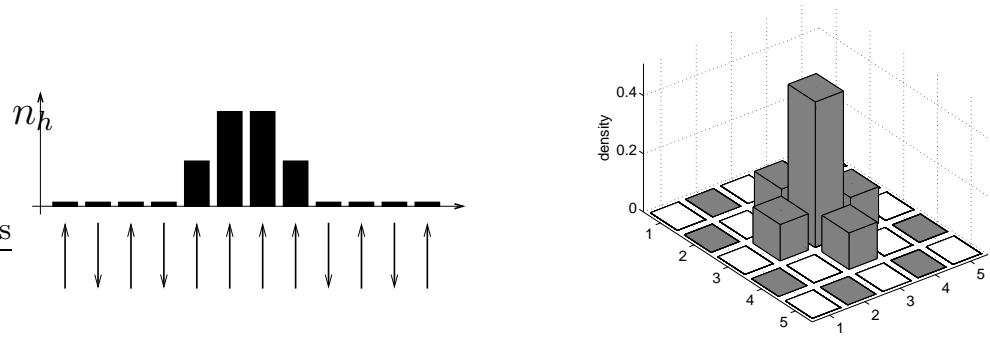

Fig. 6. Toy model for FM polarons in one (left) and two (right) dimensions. Height represents hole density. In $1 \mathrm{D}$, a FM domain of $L_{f}=4$ lattice sites is embedded in an AFM background, in 2D one spin is flipped from the perfect AFM. A single hole is localized in the FM domain giving rise to the depicted hole density (different from the schematic shape in Fig. 4 of Ref. [26]). Left from Ref. [24], right from Ref. [25].

principal effect of the fluctuations is a finite bandwidth for the AFM at half filling, their amount was therefore fitted to yield the same width for the AFM band as the MC data, see Fig. 8.

Because the FM wells in which the hole can move are so small, they give rise to only a few well separated signals in the spectral density. Figure 7 shows the spectral density and the density of states for one, two and three holes in one dimension. On sees a broad band in the center which comes from holes moving in the imperfect AFM background. Separated from this central band by a (mirror) pseudogap are dispersionless states from the FM polarons at $\omega \simeq \pm 1.5$. The weights of these signals increase upon the introduction of more holes.

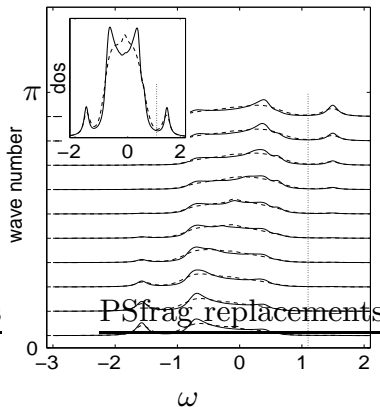

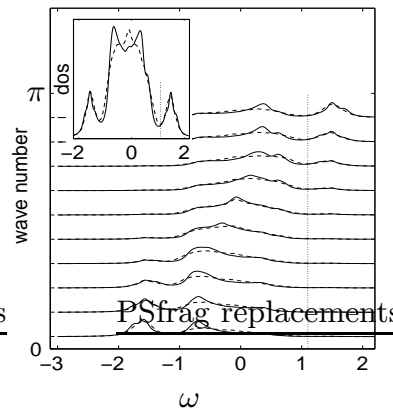

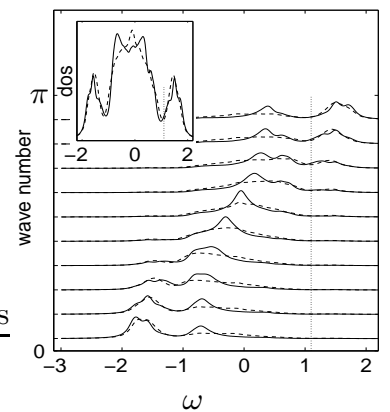

Fig. 7. Spectral density for $N_{h}=1$ hole, $N_{h}=2$ holes $N_{h}=3$ holes, corresponding to 1, 2 and 3 polarons in one dimension. Dashed lines: MC data, solid: generalized UHA. Parameters as in Fig. 4. From Ref. [24].

Figure 8 shows the spectral density for the two dimensional model with 6 and 20 holes and compares it to the data for the toy model with added random 

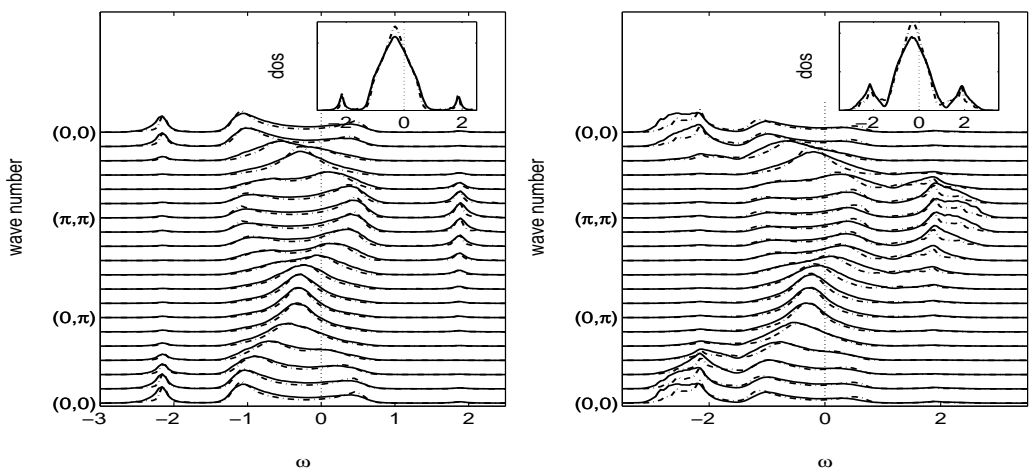

Fig. 8. Spectral density for $J^{\prime}=0.02, \beta=50, J_{\mathrm{H}}=6$ on a $12 \times 14$ lattice: left: 6 holes $(x \approx 0.035)$; right: 20 holes $(x \approx 0.12)$. Solid lines are the unbiased MC data, dashed lines the simplified polaron model.

fluctuations. There is again a broad central band from the AFM featuring a mirror band due to the doubling of the unit cell and again the polaronic states separated by a pseudogap. There is strong correspondence of the unbiased MC data to the simplified model for both the one- and the two dimensional system.

The pseudogap, which is also observed in experiments [27-30], can easily be explained by the few well separated eigenenergies of the holes trapped in the small polarons. In a phase separation scenario with larger FM regions, additional states would fill the gap between the AFM band and the polaron states, in contrast to experimental results and the $\mathrm{MC}$ data.

\subsection{Phase diagram in 2D}

Although $0<J^{\prime}<0.1$ is by far the smallest parameter of the Hamiltonian, it has a considerable effect; especially at low carrier density, when the kinetic energy is small. While we observed nearly independent polarons for $\beta=50$ and $J^{\prime}=0.02$, they tend to form larger clusters and eventually phase separation for decreasing $J^{\prime}$ and avoid each other for larger $J^{\prime}$. The reason for this effect is the stabilization of the AFM background by $J^{\prime}$.

In order to determine the phase boundary between the polaronic regime and phase separation, we chose the filling, at which the nearest AFM signal (at the distance $r^{2}=5$ ) in the dressed core-spin correlation (18) became ferromagnetic. This criterion is somewhat arbitrary and the transition is not sharp, polarons rather coexisting with larger clusters.

For large doping, $J^{\prime}=0.05$ destroys ferromagnetism and leads to the so called flux phase around half filling $[11,12,31,32]$. In the phase diagram, one sees a small window in parameter space for phase separation, but for realistic parameters $J^{\prime}>0.01$, polarons dominate, especially at small doping. 


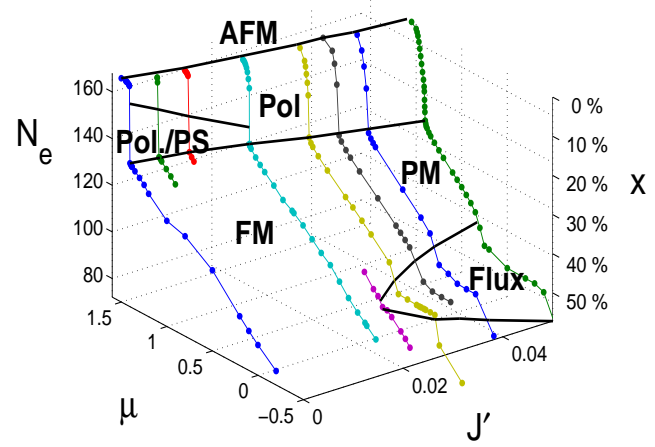

Fig. 9. Electron number $N_{e}$ as a function of $\mu$ and $J^{\prime}$, and phase diagram for $-0.5<\mu<1.6,0 \leq J^{\prime} \leq 0.05,0 \leq x \leq 0.6$ (i. e. filled to $40 \%$ filled lower Kondo band), $J_{\mathrm{H}}=6, \beta=50$ on a $14 \times 12$-lattice. "Pol.": polaronic regime, "Pol./PS": mixture of both polarons and larger ferromagnetic clusters, "FM": ferromagnet, "AFM": antiferromagnet, "PM": regime without magnetic structure, "Flux": Flux phase.

\section{Summary}

An effective spinless fermion model (ESF) was derived from the FM Kondo lattice model (1) with classical core spins and for $J_{H} \gg t$, leading to the ESF Hamiltonian (7). It allows the treatment of finite $J_{H}$ with the same numerical effort as the $J_{H} \rightarrow \infty$ approximation, but gives better correspondence to the original model. A further simplification was achieved by treating the fluctuating core spins by a uniform hopping strength (UHA). With this much simpler model (13), we obtained the Curie temperature for the 3D model with one itinerant orbital in accordance with experimental values.

By unbiased MC simulations for the ESF model (7) with non-degenerate conduction band in one and two dimensions, we found that ferromagnetic polarons are the reason for features previously attributed to phase separation. This polaronic behavior is enhanced by larger $J^{\prime}>0.02$. A phase diagram was obtained for the 2 dimensional case.

\section{References}

1. T.A. Kaplan and S.D. Mahanti, editors. Physics of Manganites. Kluwer Academic/ Plenum Publishers, New York, Boston, Dordrecht, London, Moscow, 1. edition, 1998.

2. E. Dagotto, T. Hotta, and A. Moreo. Colossal magnetoresistant materials: the key role of phase separation. Phys. Rep., 344(1-3):1-153, 2001.

3. F. Wang and D. P. Landau. Determing the density of states for classical statistic models: A random walk algorithm to produce a flat histogram. Phys. Rev. E, 64(5), 2001. 
4. N. Furukawa. Thermodynamics of the double exchange systems. In T.A. Kaplan and S.D. Mahanti, editors, Physics of Manganites, New York, 1998. Kluwer Academic Publisher.

5. E. Dagotto, S. Yunoki, A. L. Malvezzi, A. Moreo, J. Hu, S. Capponi, D. Poilblanc, and N. Furukawa. Ferromagnetic Kondo model for manganites: Phase diagram, charge segregation, and influence of quantum localized spins. Phys. Rev. B, 58(10):6414-27, 1998.

6. D. Meyer, C. Santos, and W. Nolting. Quantum effects in the quasiparticle structure of the ferromagnetic Kondo lattice model. J. Phys. Condens. Matter, 13:2531, 2001.

7. W. Müller and W. Nolting. Temperature-dependent quasiparticle band structure of the ferromagnetic semiconductor eus. Phys. Rev. B, 66:085205, 2002.

8. D. J. Garcia, K. Hallberg, C. D. Batista, S. Capponi, D. Poilblanc, M. Avignon, and B. Alascio. Charge and spin inhomogeneous phases in the ferromagnetic Kondo lattice model. Phys. Rev. B, 65:134444, 2002.

9. D. R. Neuber, M. Daghofer, R. M. Noack, H. G. Evertz, and W. von der Linden. Ferromagnetic polarons in the one-dimensional ferromagnetic Kondo model with quantum mechanical s=3/2 core spins. cond-mat/0501251, 2005.

10. A. Auerbach. Interacting Electrons and Quantum Magnetism. Springer-Verlag, New York, Berlin, Heidelberg, 1. edition, 1994.

11. H. Aliaga, B. Normand, K. Hallberg, M. Avignon, and B. Alascio. Island phases and charge order in two-dimensional manganites. Phys. Rev. B, 64:024422, 2001.

12. M. Yamanaka W. Koshibae and S. Maekawa. "flux state in the double-exchange model. Phys. Rev. Lett., 81:5604, 1998.

13. S. Yarlagadda and C. S. Ting. Mean-field approach to charge, orbital, and spin ordering in manganites. Int. J. Mod. Phys. B, 15(19-20):2719-26, 2001.

14. Shun-Qing Shen and Z. D. Wang. Phase separation and charge ordering in doped manganite perovskites: Projection perturbation and mean-field approaches. Phys. Rev. B, 61(14):9532-41, 2000.

15. W. Koller, A. Prüll, H. G. Evertz, and W. von der Linden. Effective spinless fermions in the strong-coupling Kondo model. Phys. Rev. B, 66:144425, 2002.

16. J. van den Brink and D. Khomskii. Double-exchange via degenerate orbitals. Phys. Rev. Lett., 82(5):1016-19, 1999.

17. W. Koller, A. Prüll, H. G. Evertz, and W. von der Linden. Uniform hopping approach to the FM Kondo model at finite temperature. Phys. Rev. B, 67:104432, 2003.

18. S. Yunoki, J. Hu, A. L. Malvezzi, A. Moreo, N. Furukawa, and E. Dagotto. Phase separation in electronic models for manganites. Phys. Rev. Lett., 80(4):845-8, 1998.

19. A. Urushibara, Y. Moritomo, T. Arima, A. Asamitsu, G. Kido, and Y. Tokura. Insulator-metal transition and giant magnetoresistance in $\mathrm{La}_{1-x} \mathrm{Sr}_{x} \mathrm{MnO}_{3}$. Phys. Rev. B, 51(20):14103-14109, 1995.

20. A. O. Sboychakov, A. L. Rakhmanov, K. I. Kugel, M. Yu. Kagan, and I. V. Brodsky. Tunneling magnetoresistance of phase-separated manganites. J. Exp. Theor. Phys., 95:753, 2002.

21. A. Moreo, S. Yunoki, and E. Dagotto. The phase separation scenario for manganese oxides. Science, 283:2034, 1999.

22. D. M. Edwards. Ferromagnetism and electron-phonon coupling in the manganites. Adv. Phys., 51(5):1259-1318, 2002. 
23. T. Wu, S. B. Ogale, J. E. Garrison, B. Nagaraj, A. Biswas, Z. Chen, R. L. Greene, R. Ramesh, T. Venkatesan, and A. J. Millis. Electroresistance and electronic phase separation in mixed-valent manganites. Phys. Rev. Lett., 86(26):59986001, 2001.

24. W. Koller, A. Prüll, H. G. Evertz, and W. von der Linden. Magnetic polarons in the FM Kondo model. Phys. Rev. B, 67:174418, 2003.

25. M. Daghofer, W. Koller, H. G. Evertz, and W. von der Linden. Polaronic aspects of the two-dimensional Kondo model. J. Phys.: Condens. Matter, 16:5469, 2004.

26. A. Moreo, S. Yunoki, and E. Dagotto. Pseudogap formation in models for manganites. Phys. Rev. Lett., 83:2773-2776, 1999.

27. D. S. Dessau, T. Saitoh, C. H. Park, Z. X. Shen, P. Villella, N. Hamada, Y. Moritomo, and Y. Tokura. K-dependent electronic structure, a large "ghost" fermi surface, and a pseudogap in a layered magnetoresistive oxide. Phys. Rev. Lett., 81(1):192, 1998.

28. T. Saitoh, D. S. Dessau, Y. Moritomo, T. Kimura, Y. Tokura, and N. Hamada. Temperature-dependent pseudogaps in colossal magnetoresistive oxides. Phys. Rev. B, 62(2):1039-43, 2000.

29. Y.-D. Chuang, A. D Gromko, D. S. Dessau, T. Kimura, and Y. Tokura. Fermi surface nesting and nanoscale fluctuating charge/orbital ordering in colossal magnetoresistive oxides. Science, 292:1509, 2001.

30. J.H. Park, C. T. Chen, S-W. Cheong, W. Bao, G Meigs, V. Chakarian, and Y. U. Idzerda. Electronic aspects of the ferromagnetic transition in manganese perovskites. Phys. Rev. Lett, 76:4215, 1996.

31. D. F. Agterberg and S. Yunoki. Spin-flux phase in the Kondo lattice model with classical localized spins. Phys. Rev. B, 62:13816, 2000.

32. M. Daghofer, W. Koller, W. von der Linden, and H. G. Evertz. Single particle spectrum of the flux phase in the fm kondo model. Physica B, 359-361C:804, 2005. 